\title{
ARTIGOORIGINAL
}

\section{PAImagem: uma ferramenta para quantificação de áreas em imagens de microbiologia}

\section{PAImagem: a tool for image area quantification in microbiology}

\author{
Cassandro Davi Emer ${ }^{\oplus}, 1$, Angelo Gava ${ }^{(2,2,3}$, Paulo Ricardo Cechelero Villa ${ }^{(\bullet, 1}$ \\ ${ }^{1}$ Computação Aplicada, IFRS, Campus Veranópolis, ${ }^{2}$ IFRS, Campus Bento Gonçalves, ${ }^{3}$ Programa de Pós-Graduação \\ em Microbiologia Agrícola e do Ambiente, UFRGS. \\ *cassandro@gmail.com
}

Recebido: 05/05/2020. Revisado: 15/07/2020. Aceito: 20/07/2020.

\section{Resumo}

Mensurar o crescimento de microrganismos é de grande importância na microbiologia. A metodologia para avaliação de crescimento, embora simples, possui algumas desvantagens, como ser um processo repetitivo, demandar elevado tempo, e sobretudo, seus resultados dependerem da observação do analista e tratarem crescimentos irregulares de forma subjetiva. Desta forma, objetivou-se desenvolver e validar uma aplicação que visa auxiliar na quantificação de áreas em microbiologia por meio de imagens digitais, a fim de disponibilizar uma alternativa ao método convencional. PAImagem foi desenvolvido para intermediar o processamento de imagens tomadas em condições controladas, com pontos referenciais, de modo a compensar a perspectiva e determinar escala. O utilitário foi concebido a partir de um design dirigido por objetivos, com critérios de qualidade da Interface Humano-Computador. A validação da ferramenta deu-se a partir do acompanhamento do crescimento do fungo Glomerella cingulata, realizado manualmente e pela captura de imagens. Dados foram adquiridos a cada $24 \mathrm{~h}$ por 14 dias, avaliando condições normais e adversas de crescimento. Através do coeficiente de Pearson, foi observada uma elevada correlação $(R=0,99)$ entre a área calculada pela medida por régua e a verificada pelo PAImagem. A presente ferramenta mostrou ser de grande validade para o uso em pesquisas envolvendo microrganismos, otimizando o tempo de ensaios em laboratórios.
\end{abstract}

Palavras-Chave: Crescimento microbiológico; Estimativa de área em imagem; Processamento de imagem

\section{Abstract}

Measuring microbial growth is a major subject in microbiology. The growth measurement methodology, although simple, has some disadvantages, such as being a repetitive process, requiring prolonged times, and above all, its results depend on the analyst's observation, and irregular growths are approached subjectively. In this way, the objective was to develop and validate an application that aims to assist in the quantification of areas in microbiology through digital images, in order to provide an alternative to the conventional method. PAImagem was developed to assist the processing of images taken under controlled conditions, with reference points, in order to compensate the perspective and determine scale. The tool was conceived from a design directed by objectives, with Human-Computer Interface quality criteria. The validation of this tool was based on monitoring the growth of Glomerella cingulata fungus, carried out with manual measurement and image capture. Data were acquired every 24 hours for 14 days, assessing normal and adverse growth conditions. Through the Pearson coefficient, a high correlation $(\mathrm{R}=0.99)$ was observed between the area calculated from the measurement using a ruler, and the one verified by PAImagem. The present tool proved to be of great value for use in researches involving microorganisms, optimizing the time of trials in laboratories. 


\section{Introdução}

Determinar a suscetibilidade e capacidade de crescimento de microrganismos mediante diferentes parâmetros e exposição a compostos é de grande importância na microbiologia, não apenas para o entendimento da resposta dos microrganismos frente a compostos e condições específicas (Satapathy and Beura, 2019, Wang et al., 2019, Ebrahimzadeh and Abrinbana, 2019, Cáceres Candia, 2020, Kim et al., 2010, Zhang et al., 2017), mas também para investigar o desenvolvimento de resistência (Ghini and Kimati, 2000, Leroch et al., 2011, Hu et al., 2016)

Existem vários métodos para determinar a capacidade de crescimento e a suscetibilidade antimicrobiana, incluindo difusão em disco, diluição em ágar ou micro-diluição em caldo (Balouiri et al., 2016). Todas essas técnicas são executadas em condições padronizadas (meios, temperaturas, tempos de incubação, entre outras), a fim de obter resultados interpretáveis, reprodutíveis e comparáveis.

Para avaliar o crescimento micelial ou da área de inibição por disco-difusão, uma amostra do microrganismo, de tamanho uniforme, é inserida na placa de Petri para crescer em ambiente controlado. Para avaliar o crescimento, o monitoramento é comumente feito pela análise visual, por meio de mensurações utilizando-se de régua ou paquímetro, desta forma, acompanhando periodicamente o desenvolvimento do microrganismo (Martins et al., 2015, Kai et al., 2019). Esta técnica tem baixíssimo custo de material, não sendo necessário extrair amostras das placas, pré-tratamentos ou procedimentos analíticos, não requerendo reagentes, equipamentos ou sensores sofisticados. Também não existe a necessidade de contato físico com o patógeno, evitando contaminações. Porém, apesar do processo de acompanhamento ser simples, existem certas desvantagens de ordem prática (Kai et al., 2019), enumeradas:

i. Em pesquisa, o número de amostras cultivadas simultaneamente, de maneira geral, é alto, dada a necessidade de repetições para validação estatística dos dados. $\mathrm{O}$ acompanhamento de um número elevado de amostras torna o processo repetitivo e tedioso; ii. A demanda de tempo para ponderação das áreas em placas será proporcional ao número de amostras, o que exige disponibilidade de tempo;

iii. Os microrganismos, em algumas condições, podem levar ciclos de desenvolvimentos longos até atingirem o crescimento máximo suportado pela placa, fazendo com que as atividades se tornem cansativas; iv. A avaliação dos dados coletados demanda tempo, sendo que, em determinadas situações, caso houvesse a pronta visualização dos dados adquiridos, seria possível a correção de desvios ou adequação do cenário de uma investigação, diminuindo custos e tempo;

v. A aferição de zonas de inibição ou crescimento pode ser altamente dependente do observador, sendo possível obter resultados distintos pela simples troca do analista, seja pela experiência ou características pessoais; vi. Muitos microrganismos desenvolvem áreas de crescimento irregulares, o que dificulta muito a medição e a torna mais subjetiva.

Sendo assim, este trabalho apresenta o desenvolvimento e validação de uma aplicação que visa auxiliar a quantificação de áreas em microbiologia por meio de imagens digitais. Tal trabalho é de grande valor na pesquisa de microrganismos, sendo uma alternativa rápida e eficaz à medição tradicional, via medição com réguas ou paquímetros.

\section{Trabalhos relacionados}

Existem alguns aplicativos com usabilidades similares ou relacionadas às implementadas no aplicativo PAImagem. A fim de clarificar as funcionalidades, pontos positivos e negativos de cada uma das ferramentas são apresentados, de forma comparativa quando cabível:

i. AFSoft: utilitário gratuito, disponível para Windows, concebido para a determinação de área foliar. Assim como o PAImagem, pode ser utilizado em outras áreas, porém, não suporta o ajuste automático da perspectiva, sendo necessária a realização de prétratamento das imagens e calibração manual para cada imagem ou lote;

ii. CellProfiler: aplicação gratuita, para Windows e MacOS, criada para análise quantitativa em imagens biológicas. Apesar da alta aplicabilidade e recursos disponíveis, a ferramenta foi desenvolvida para lidar, principalmente, com imagens geradas a partir de aparatos específicos, o que dificulta a utilização de fotografias não padronizadas;

iii. ImageJ: utilitário gratuito, disponível para MacOS, Linux e Windows. Mais do que um utilitário, tratase de uma biblioteca bastante completa de processamento de imagens que, apesar de realizar inúmeras operações, necessita que o usuário possua um elevado nível de conhecimento técnico para a utilização, já que o algoritmo de identificação é determinado manualmente;

iv. OpenCFU: ferramenta gratuita, com interface simples e amigável, disponível em versões para Linux e Windows. Embora também processe imagens de placas de microbiologia, o intuito principal da ferramenta OpenCFU é a identificação de pontos de contraste, de forma a automatizar a contagem de unidades formadoras de colônia.

Muito embora existam ferramentas com finalidade parecida, nenhuma delas apresenta as características do PAImagem em relação à flexibilidade na obtenção das imagens, uma vez que o próprio usuário, através do processo interativo, irá realizar as definições necessárias para o funcionamento da ferramenta, sem requerimento de padronização da orientação, distância e perspectiva na obtenção das fotografias, e de forma independente do dispositivo de captura de imagens. 


\section{Implementação}

A aplicação PAImagem foi desenvolvida utilizando a linguagem de programação Java. Foi primariamente direcionada para a plataforma Windows, mas pelo caráter da linguagem, há a possibilidade de ser portado para outras plataformas. A ferramenta tem dependência de uma biblioteca de terceiros amplamente usada para software de visão computacional e aprendizado de máquina, chamada OpenCV (OpenCV, 2019), que possui vários algoritmos para manipulação de imagens. Além disso, o PAImagem usa a biblioteca Apache POI (Apache Software Foundation, 2020), que permite a exportação dos resultados em planilhas no formato do Microsoft Excel.

A concepção do programa deu-se através do design dirigido por objetivos, em que o analista projeta uma solução que auxilie usuários para que atinjam seus objetivos, atendendo os requisitos técnicos e de negócio. A elaboração da interface foi desenvolvida tendo em vista critérios ergonômicos, com o emprego da Lei de Hick-Hyman e Lei de Fitts (Seow, 2005), além de levar em conta os princípios da psicologia cognitiva.

As imagens utilizadas como fonte de dados para o programa podem ser capturadas com qualquer dispositivo de câmera, seja câmera digital, smartphone ou tablet. Não existe uma padronização específica para a coleta das imagens, o único requerimento é que haja um quadro demarcado por quatro pontos referenciais simétricos e de distância entre centros conhecida, usualmente circulares (mas não necessariamente). Os pontos referenciais servem tanto para o dimensionamento da área em unidades físicas de grandeza $\left(\mathrm{cm}^{2}\right)$, como para a transformação geométrica da perspectiva. Em relação à luminosidade na obtenção das imagens, embora a uniformidade não seja uma exigência, é interessante do ponto de vista da homogeneidade das amostras para o processamento em lotes, tornando o uso das configurações mais amplo.

Existem quatro conceitos principais dentro do PAImagem: Área de interesse, Filtro de processamento, Arranjo de recorte e Configuração. Uma Área de interesse é a representação de um segmento retangular ou elíptico, vinculado a um referencial absoluto ou relativo, que deve ser considerado individualmente pelo programa, identificado por uma cor e nome customizáveis. Já o Filtro de processamento é um intervalo entre cores com uma opção de tolerância que, quando no momento do processamento da imagem, será utilizado para determinar a área que deve ser computada. O Arranjo de recorte é a configuração de quatro áreas identificáveis nas imagens, usualmente circulares, determinados por um intervalo de cor preferencialmente estreito e de distância entre centros conhecida. Finalmente, a Configuração corresponde ao conjunto de informações necessárias para a realização de uma análise, incluindo o formato de identificação, opções de ajuste de imagens (rotação, redimensionamento e efeito de luminosidade), o seu Arranjo de recorte, Áreas de interesse e Filtros de processamento.

\subsection{Determinação do Arranjo de recorte}

Durante o processo interativo, no momento em que o usuário inicia a elaboração de uma nova Configuração utilizando um arquivo de imagem, além da determinação das preferências em relação ao processamento das imagens, ele deve criar um Arranjo de recorte. A partir deste arranjo, será realizado o corte da área interna dos delimitadores, o que além de estreitar o processamento à área útil, permite a correção da perspectiva (Fig. 2), no caso de utilização de fotografias obtidas de forma angulada em relação à superfície das placas.

A transformação geométrica de quatro pontos se dá através de um processamento pixel a pixel, em que é realizada a deformação da grade e mapeamento desta para a imagem de destino, ou seja, para cada pixel da imagem de destino, são computas as coordenadas do correspondente pixel "doador" na imagem de origem.

A determinação do Arranjo de recorte envolve a determinação das distâncias entre os centros das figuras, chamadas delimitadores na solução de IHC (Interface Humano-Computador), e seleção de um intervalo de cores que permita a sua caracterização. A Fig. 4 representa o momento em que o usuário, após a determinação do intervalo de cores, visualiza a resposta da aplicação pelo destaque das áreas encontradas e seus pontos centrais, bem como uma tabela com suas áreas para que, no caso de interferência, possa seja aplicada uma restrição por área mínima.

No instante em que a aplicação encontra quatro pontos distintos que permitem a realização do recorte, o usuário é informado e a etapa de elaboração do Arranjo de recorte é concluída.

\subsection{Criação de Filtros de processamento}

No momento em que a interação passa a contar com um Arranjo de recorte válido, a imagem referência na interface passa a ser o resultado do processo da correção da perspectiva, e as funcionalidades de manipulação de Filtros de processamento são habilitadas dentro do editor de Configurações.

O diálogo da edição de configuração passa a apresentar a imagem já cortada e sem perspectiva (Fig. 2), conforme os atributos selecionados no diálogo da determinação do Arranjo de recorte (ou delimitadores), como ilustrado na Fig. 3. Nesta mesma imagem, é possível visualizar diversas entidades representando os filtros de cor, ilustrados pelos quadros coloridos que caracterizam os intervalos de cores, localizadas na parte inferior da coluna à esquerda.

A determinação do intervalo de cores para cada um dos Filtros de processamento é realizada de forma interativa, sendo o usuário apresentado à imagem de referência com um "alvo" que deve ser arrastado com o ponteiro do mouse sobre as áreas com as cores que são de interesse para o intervalo sendo determinado. 0 "alvo" apresenta um zoom local, como uma espécie de lupa, para que permita um posicionamento preciso do centro. Ao atingir uma área de pertinente ao filtro, o usuário aciona o botão "Adicionar" ou pressiona a tecla de ação no teclado (Enter) tendo o botão em foco. Os 


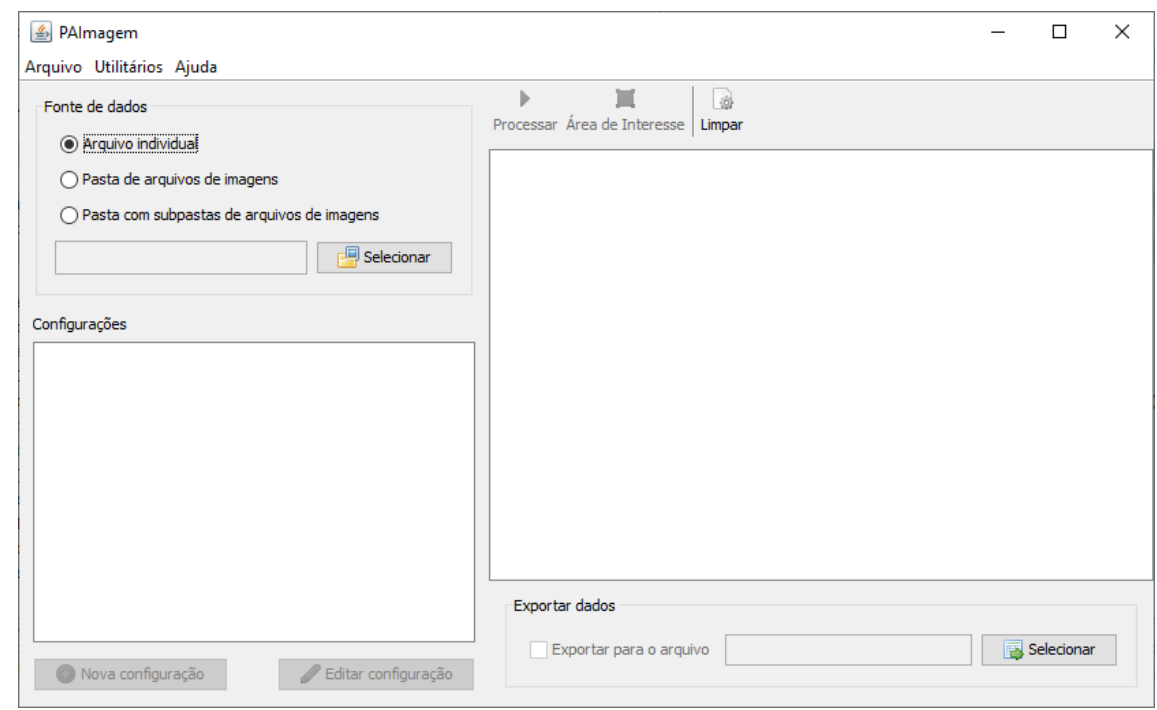

Figura 1: Tela inicial da aplicação PAImagem

limites superiores e inferiores de cores são computados pela aplicação a partir do conjunto de valores de cores dos pontos selecionados, acrescidos da margem determinada pelo valor definido como "tolerância".

\subsection{Processamento}

Dentro da tela da edição da configuração (Fig. 3), na parte superior direita, o usuário tem a possibilidade de realizar o processamento para cálculo da área total (operação binária $\mathrm{OR}$ ) do conjunto de Filtros de processamento, da mesma maneira que seria realizado pela tela inicial (Fig. 1), porém com um outro nível de detalhamento na metalinguagem, servindo assim, para o usuário validar o andamento da elaboração da Configuração durante o processo interativo.

Quando existem Áreas de interesse determinadas pelo usuário, o processamento realizado dentro da interface de edição da Configuração irá resultar em uma tabela com os dados computados para cada uma das Áreas de interesse, como ilustrado na Fig. 5. Repare que, a parte da área da máscara (imagem binária representando a região de interesse) computada pelo somatório dos Filtros de processamento (detalhado na Seção 3.3.1), é determinado o raio do círculo desenhado na figura da coluna "Recorte", estimado automaticamente quando a máscara possui um formato arredondado. A informação do raio, embora útil, deve ser utilizada com cautela, uma vez que o círculo envolve a área da máscara pelo seu exterior, ou seja, caso sejam comparados, no mesmo tipo de métrica e unidade, tenderá a apresentar um valor maior e menos preciso do que a área ponderada pelos filtros (máscara). Logicamente, no caso de formações irregulares, somente a área possui utilidade e, nesses casos, não é possível a determinação do raio do círculo pelo programa.

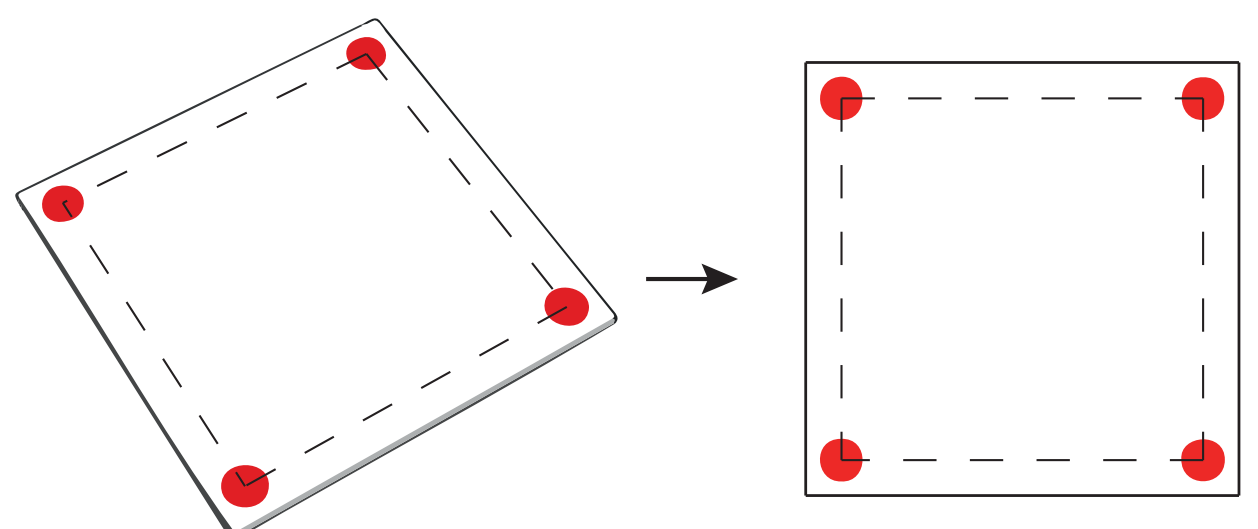

Figura 2: Ilustração da correção da perspectiva baseada nos centros dos delimitadores 


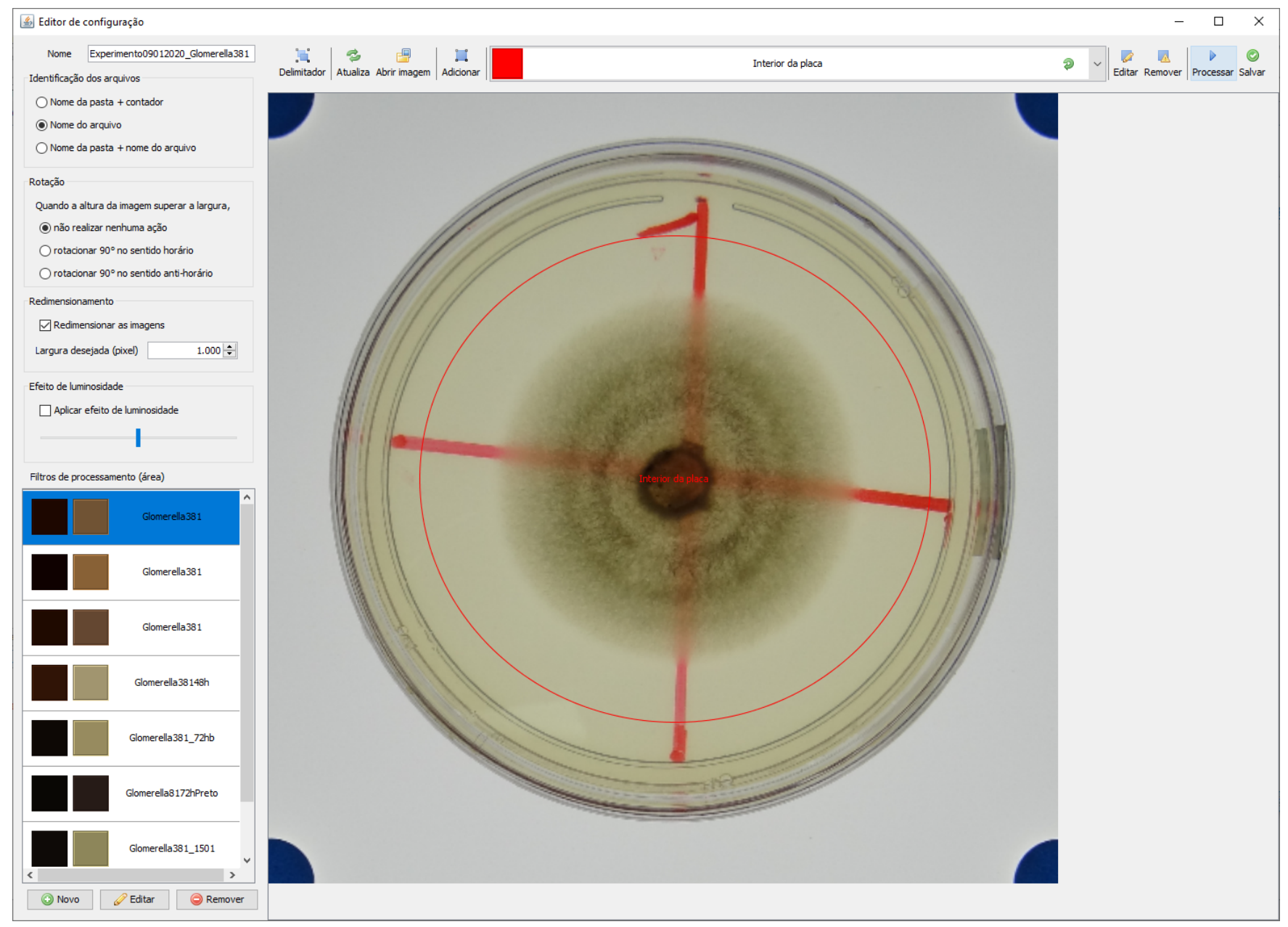

Figura 3: Interface de edição de Configuração, apresentando uma Área de interesse central, delimitada por um círculo em vermelho, e diversos Filtros de processamento 


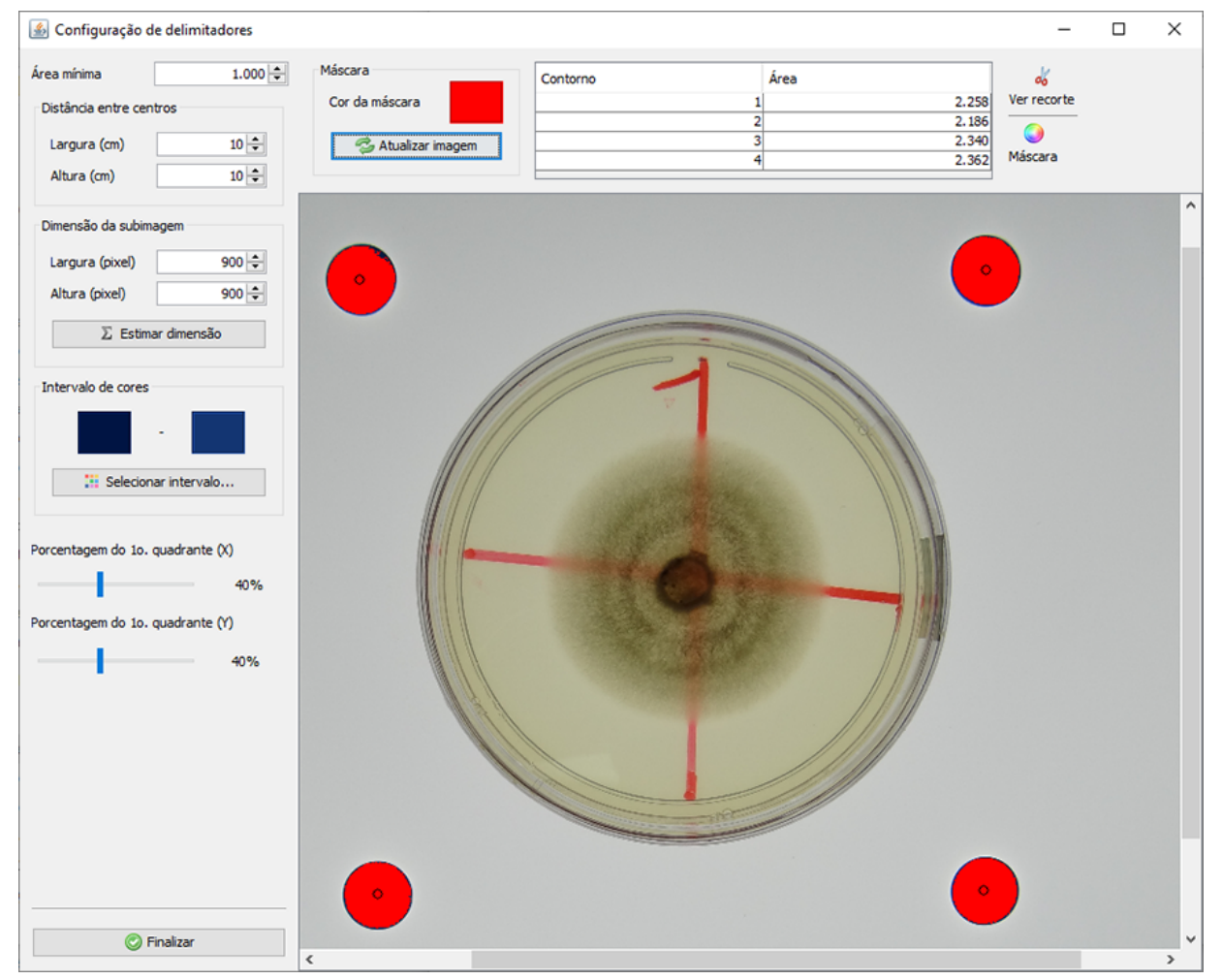

Figura 4: Tela de configuração dos delimitadores do Arranjo de recorte, destacando em vermelho as áreas delimitadoras

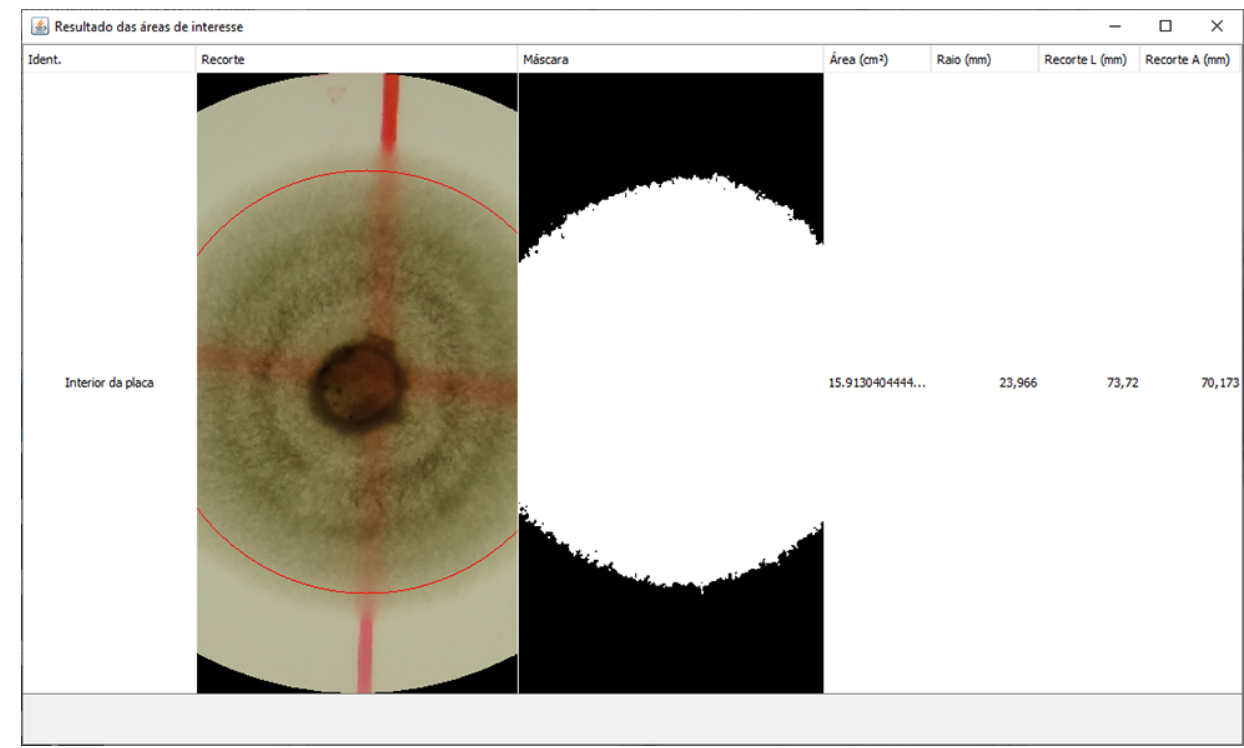

Figura 5: Diálogo apresentando os dados computados pelo processamento das Áreas de interesse determinadas pelo usuário 


\subsubsection{Criação de máscaras e cálculo de área}

A criação das máscaras é realizada individualmente através da verificação se cada um dos elementos da matriz (cada um dos pixels da imagem) estão compreendidos em um intervalo de cor (para cada um dos Filtros de processamento). O algoritmo emprega a iteração da matriz bidimensional de tamanho $i$ por $j$, através da Eq. (1).

$$
\text { Mask }_{i, j}=\operatorname{lower}_{b} \leq \operatorname{src}_{i, j} \leq \text { upper }_{b}
$$

onde:

Mask é a máscara (matriz de saída do mesmo tamanho de $s r c$ )

src é a matriz da imagem sendo processada

lower $_{b}$ é o limite inferior inclusivo (coloração mais escura)

upper $_{b}$ é o limite superior inclusivo (coloração mais clara)

Uma vez computadas as máscaras para cada um dos filtros, é calculada a disjunção lógica em bits por elemento, gerando uma silhueta da área total dos filtros. O processo é realizado repetidamente, usando o produto anterior como parâmetro de entrada do próximo cálculo. O processo pode ser representado pela Eq. (2):

$D s t=s r c_{1} \vee s r c_{2} \Rightarrow D s t=D s t \vee s r c_{3} \Rightarrow \cdots \Rightarrow D s t=D s t \vee s r c_{n}$

onde:

Dst é a máscara resultante da operação binária $\operatorname{src}_{1 . . n}$ são as máscaras criadas para cada um dos filtros de processamento

Determinada a máscara resultante, é usado o algoritmo de detecção de contornos em imagens binárias (máscaras) de Suzuki et al. (1985), resultando em um vetor de conjuntos de dados que são representações que permitem reproduzir a máscara. Usando cada um dos elementos do vetor, são computadas e somadas as áreas (em pixels) usando o teorema de Green. Finalmente, é estabelecido um fator de conversão entre a área em pixels e uma medida em centímetros quadrados, baseando-se nos dados da Configuração.

\subsubsection{Processamento de lotes}

O processamento de lotes, realizado a partir da seleção de uma pasta ou pasta com um nível de recursão na tela principal da aplicação (Fig. 1), é realizado pela varredura dos arquivos de imagem válidos (filtrados pelas extensões JPEG, JPG, GIF, TIFF, TIF e PNG) no diretório selecionado pelo usuário, classificando-os em ordem alfabética pelo nome do arquivo. A ordem em que é realizada a leitura é a mesma da inserção do dado computado, tanto na caixa de texto do andamento do processamento, como no arquivo de exportação, quando selecionado.

\subsubsection{Exportação de dados}

A exportação dos dados é realizada utilizando a biblioteca Apache POI (Apache Software Foundation, 2020), no formato do utilitário de planilhas amplamente utilizado, Microsoft Excel. A parte dos campos de texto e numéricos, contendo as informações de identificação do arquivo e área computada, são também incorporadas imagens dos recortes e máscaras que, mesmo em versões de baixa resolução, permitem uma validação, por parte do usuário, da interpretação da área ponderada pelo programa.

\subsection{Persistência}

Dentro da aplicação, a realização de persistência de uma Configuração se dá pela gravação em disco de um arquivo XML em uma pasta dedicada, junto ao arquivo executável do programa. O arquivo XML é nomeado conforme a etiqueta de descrição da Configuração, incorporado um numeral no caso de o arquivo já existir, ou com um valor numérico (a hora do sistema no formato Unix time em mili segundos) quando a etiqueta estiver sem nenhum valor preenchido.

Os arquivos XML são intercambiáveis e manualmente editáveis, embora alguns atributos, pelo caráter técnico, sejam customizáveis muito mais facilmente pela solução de IHC.

\section{Validação}

Foi realizado um experimento de acompanhamento de crescimento fúngico, a fim de validar a consistência das leituras realizadas pela aferição com régua e as realizadas pelo utilitário PAImagem a partir de imagens obtidas por câmera digital.

\subsection{Materiais e métodos}

O experimento foi desenvolvido no laboratório de Fitopatologia do Instituto Federal de Educação, Ciência e Tecnologia do Rio Grande do Sul - Campus Bento Gonçalves (IFRS-BG). O fungo utilizado para a validação foi Glomerella cingulata (IFBG-1406 - SisGen $n^{\circ}$ A7F3DBF), obtido da Micoteca do Laboratório de Fitopatologia, sendo este o agente causador da podridão da uva madura.

Para o crescimento, foi realizado um cultivo em ágar batata-dextrose (BDA) por 7 dias para a retirada de discos de micélio com $8 \mathrm{~mm}$ de diâmetros. Os mesmos foram, então, transferidos para o centro de novas placas de Petri contendo o mesmo meio (BDA). As colônias fúngicas foram, então, cultivadas e o diâmetro da colônia foi medido a cada $24 \mathrm{~h}$ e em duas direções perpendiculares. No mesmo momento em que eram realizadas as mensurações por régua, as placas de $\mathrm{Pe}-$ tri eram fotografadas. $\mathrm{O}$ experimento foi considerado concluído quando o fungo cresceu por toda a extensão da placa, tendo o crescimento perdurado um período de 14 dias e as placas mantidas a $25 \pm 2^{\circ} \mathrm{C}$ em escuro contínuo. 
No mesmo laboratório, foi implantado um mini estúdio que consistia em uma câmera fotográfica Carl Zeiss Cyber Shot (Sony) instalada sobre um tripé com coluna horizontal (Manfrotto). As fotografias das placas de Petri contendo o micélio do fungo foram obtidas em fundo branco contendo um plafon de LEDs, sendo demarcadas por 4 etiquetas circulares com áreas e distâncias entre centros conhecidas.

Condições normais e adversas de crescimento foram simuladas para a validação, através da incorporação de diferentes antifúngicos e diferentes doses no preparo dos meios. Desta forma, foram avaliados 10 diferentes condições com 4 repetições cada. Ao todo, resultaram 560 pares de dados (40 pontos experimentais por 14 dias) para avaliar a correlação entre a leitura por régua e a calculada pelo PAImagem através da imagem. Um mesmo analista realizou todas as medidas, tanto por régua quanto por imagem. O tempo médio diário para verificação do crescimento por régua relatado pelo analista foi de aproximadamente 90 minutos, enquanto o tempo de tomada de imagens diário foi de aproximadamente 15 minutos.

\subsection{Análise de Correlação}

A análise de correlação foi realizada através do coeficiente de correlação de Pearson em função da área calculada pela medida realizada com régua (a partir do diâmetro do crescimento, este sendo a média das duas medidas perpendiculares) e a área de crescimento do fungo computado através da ferramenta PAImagem. $\mathrm{O}$ coeficiente de correlação de Pearson mede o grau de associação entre duas variáveis numéricas (Benesty et al., 2009, Zhou et al., 2016). Este coeficiente varia de -1 a 1 , e quanto mais próximo estiver de 1 ou -1 , mais forte é a associação. Quanto mais próximo de zero estiver o coeficiente, mais fraca é a relação entre as duas variáveis (Instituto Nacional de Metrologia, 2003, Adler and Parmryd, 2010). O coeficiente negativo expressa uma relação inversa entre as duas variáveis.

As medidas das primeiras $48 \mathrm{~h}$ foram desconsideradas para a análise de correlação, uma vez que não houve crescimento em nenhuma placa. As análises foram realizadas no software $R$ versão 3.6.3 (R Core Team, 2019). O gráfico foi elaborado utilizando o pacote ggpubr (Kassambara, 2019).

\subsection{Resultados}

Foi observada uma alta correlação entre as áreas calculadas a partir da medida com régua e as verificadas através do software (Fig. 6). O coeficiente de Pearson (R) calculado foi de 0,99 , e quanto mais próximo de 1 estiver o valor de R, mais forte é a proximidade das medidas relacionadas (Zhou et al., 2016).

É válido lembrar que, no caso do experimento realizado, o crescimento do micélio fúngico era circular e uniforme, o que permitiu a transformação da medida linear realizada manualmente em uma área, através da fórmula da área da circunferência. Destaca-se que a ponderação da área possui grande importância para

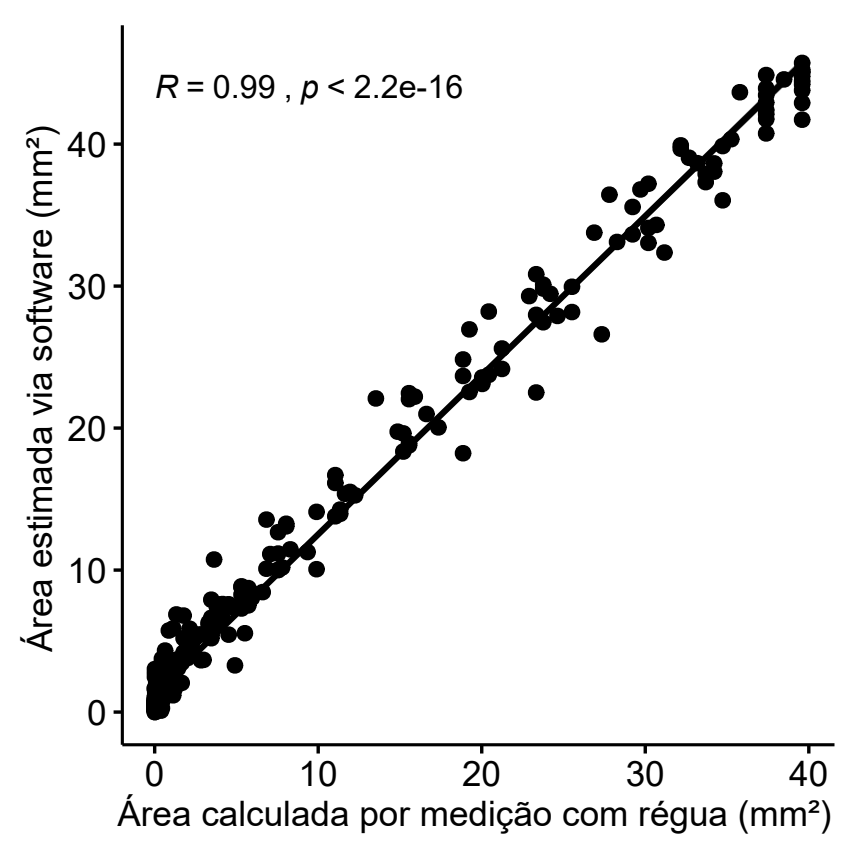

Figura 6: Gráfico de correlação das áreas entre medição manual e pela aplicação

os experimentos envolvendo microrganismos que não apresentam um formato de crescimento regular. Estudos recentes envolvendo processamento de imagem concluíram que este tipo de aplicação pode reduzir o tempo de análise e elevar a precisão, principalmente em casos em que o fungo cresce de forma irregular (da Silva et al., 2015, Kai et al., 2018, 2019).

\section{Considerações finais}

Neste artigo, apresentamos o PAImagem, um aplicativo Java projetado para medir zonas de crescimento de microrganismos em imagens de placas capturadas com qualquer dispositivo com câmera, incluindo câmeras digitais, smartphones e tablets. PAImagem é uma ferramenta focada na experiência do usuário, leve e gratuita. Além disso, a validação do software conduzida neste trabalho demonstra que o PAImagem pode ser considerado para uso em experimentos.

Como trabalho adicional, algumas melhorias estão sendo consideradas para flexibilizar o PAImagem, como lidar com antibiogramas e placas de microdiluição, se tornando uma alternativa gratuita à soluções de hardware e software comerciais. Atualmente, o PAImage pode ser baixado em qualquer PC com Windows e Java 8 ou superior instalados, a partir de www. paimagem.org.

\section{Referências}

Adler, J. and Parmryd, I. (2010). Quantifying colocalization by correlation: the pearson correlation coefficient is superior to the mander's overlap co- 
efficient, Cytometry Part A 77(8): 733-742. https: //doi.org/10.1002/cyto.a.20896.

Apache Software Foundation (2020). Apache POI - the Java API for Microsoft Documents.

Balouiri, M., Sadiki, M. and Ibnsouda, S. K. (2016). Methods for in vitro evaluating antimicrobial activity: A review, Journal of pharmaceutical analysis 6(2): 7179. https://doi.org/10.1016/j.jpha.2015.11.005.

Benesty, J., Chen, J., Huang, Y. and Cohen, I. (2009). Pearson correlation coefficient, Noise reduction in speech processing, Springer, pp. 1-4. https://doi.org/10. 1007/978-3-642-00296-0_5.

Cáceres Candia, D. P. (2020). Control de botrytis cinerea pers. en mandarina satsuma var. okitsu mediante fungicidas biológicos y químicos en huaura. Disponível em: http://repositorio.lamolina.edu.pe/handle/ UNALM/4341.

da Silva, F. O., Itako, A. T. and Júnior, J. B. T. (2015). Uso de imagens digitais para avaliar o crescimento micelial de fitopatógenos através do $\mathrm{r}, X$ Congresso Brasileiro de Agroinformática 10(1): 1-4. Disponível em: http://eventos.uepg.br/sbiagro/2015/anais/ SBIAgro2015/pdf_resumos/4/4_fernanda_oliveira_da_ silva_68.pdf.

Ebrahimzadeh, F. and Abrinbana, M. (2019). Activity of fungicide mixtures against botrytis cinerea isolates resistant to benzimidazoles, strobilurins and dicarboximides, Annals of Applied Biology 174(3): 301-312. https://doi.org/10.1111/aab.12497.

Ghini, R. and Kimati, H. (2000). Resistência de fungos a fungicidas., Jaguariúna: Embrapa Meio Ambiente, 2000. Disponível em: https://www. embrapa.br/busca-de-publicacoes/-/publicacao/ 13231/resistencia-de-fungos-a-fungicidas.

Hu, M.-J., Cox, K. D. and Schnabel, G. (2016). Resistance to increasing chemical classes of fungicides by virtue of "selection by association" in botrytis cinerea, Phytopathology 106(12): 1513-1520. https: //doi.org/10.1094/PHYTO-04-16-0161-R.

Instituto Nacional de Metrologia, N. e. Q. I. (2003). Orientações sobre validação de métodos de ensaios químicos.

Kai, P. M., de MN Soares, F. A., da Costa, R. M., Félix, J. P., de Jesus, J. M. I. and da Cunha, M. G. (2019). Measurement by images of mycelial growth of fungal colonies on petri dishes, 2019 IEEE Canadian Conference of Electrical and Computer Engineering (CCECE), IEEE, pp. 1-4. https://doi.org/10.1109/CCECE. 2019. 8861749 .

Kai, P. M. et al. (2018). Medição interativa por imagens de colônias de isolados do corynespora cassiicola em placas de petri. Disponível em: http://repositorio. bc.ufg.br/tede/handle/tede/8765.

Kassambara, A. (2019). ggpubr: 'ggplot2' Based Publication Ready Plots. R package version 0.2.3. Disponível em: https://CRAN.R-project . org/package=ggpubr.
Kim, Y. C., Lee, J. H., Bae, Y.-S., Sohn, B.-K. and Park, S. K. (2010). Development of effective environmentally-friendly approaches to control alternaria blight and anthracnose diseases of korean ginseng, European journal of plant pathology 127(4): 443-450. https://doi.org/10.1007/ s10658-010-9610-4.

Leroch, M., Kretschmer, M. and Hahn, M. (2011). Fungicide resistance phenotypes of botrytis cinerea isolates from commercial vineyards in south west germany, Journal of Phytopathology 159(1): 63-65. https : //doi.org/10.1111/j.1439-0434.2010.01719.x.

Martins, A. P., Pizolato Jr, J. C. and Belini, V. L. (2015). Avaliação automática da taxa de crescimento de colônias de leveduras incubadas em placas de petri usando plataforma móvel, Semina: Ciências Exatas e Tecnológicas 36(2): 33-46. http://dx.doi.org/ 10.5433/1679-0375.2015v36n2p33.

OpenCV (2019). Open Source Computer Vision Library.

R Core Team (2019). R: A Language and Environment for Statistical Computing, R Foundation for Statistical Computing, Vienna, Austria. Disponível em: https: //www.R-project. org/.

Satapathy, R. and Beura, S. (2019). Fungicidal management of colletotrichum gloeosporioides (penz.) causing cashew anthracnose, IJCS 7(3): 1219-1221. Disponível em: http://www.chemijournal.com/archives/ ?year $=2019 \& \mathrm{vol}=7 \&$ issue $=3 \&$ Article $\mathrm{Id}=5687 \& \mathrm{si}=\mathrm{f}$ al $\mathrm{se}$.

Seow, S. C. (2005). Information theoretic models of hci: a comparison of the hick-hyman law and fitts' law, Human-computer interaction 20(3): 315-352. http:// dx.doi.org/10.1207/s15327051hci2003_3.

Suzuki, S. et al. (1985). Topological structural analysis of digitized binary images by border following, Computer vision, graphics, and image processing 30(1): 3246. https://doi.org/10.1016/0734-189X (85) 90016-7.

Wang, X., Du, X., Nong, Y. and Shao, H. (2019). Growthinhibition of 12 fungicides against botrytis cinerea in tomato and their preventive effects in field, Pak. J. Bot 51(6): 2291-2294. http://dx.doi.org/10.30848/ PJB2019-6(32).

Zhang, S., Li, D., Song, Z., Zang, C., Zhang, L., Song, $\mathrm{X}$. and Li, S. (2017). "carbon assimilation" inspired design and divergent synthesis of drimane meroterpenoid mimics as novel fungicidal leads, Journal of agricultural and food chemistry 65(41): 9013-9021. https://doi.org/10.1021/acs. jafc. 7b03126.

Zhou, H., Deng, Z., Xia, Y. and Fu, M. (2016). A new sampling method in particle filter based on pearson correlation coefficient, Neurocomputing 216: 208-215. https://doi.org/10.1016/j.neucom.2016.07.036. 